\title{
"Choosing Wisely» pour diminuer les prestations inutiles
}

\section{Michelle Gerber ${ }^{a}$, Esther Kraft ${ }^{b}$, Christoph Bosshard ${ }^{c}$}

a lic. phil. hum., collaboratrice scientifique DDQ/ASQM; ${ }^{b}$ lic. rer. oec., cheffe de la division DDQ; ${ }^{c}$ Dr méd., vice-président de la FMH, responsable du département DDQ/ASQM

1 Par surconsommation, on entend ici une surconsommation économique et médicale. La surconsommation médicale (en anglais: overuse) désigne des "prestations avec un diagnostic ou un traitement médical qui n'est d'aucune utilité pour le patient ou dont les risques dépassent l'utilité potentielle» [1]. La surconsommation économique désigne des prestations qui sont fournies de manière inefficace ou dont l'utilite ne justifie pas les coûts [2].

2 Pour de plus amples informations, voir le document de base de l'ASQM relatif au shared decision making [3].

3 Au Canada, la démarche Choosing Wisely est très avancée avec différents documents d'information pour les patients ou avec la campagne Choosing Wisely Canada STARS lancée par des étudiants en médecine pour des étudiants en médecine (www.choosingwisely canada.org).

En se fondant sur la littérature scientifique, la division Données, démographie et qualité (DDQ) de la FMH rédige des documents de base sur différents thèmes relatifs à la qualité. Publiés dans le Bulletin des médecins suisses, ils servent de base à la FMH pour se prononcer officiellement dans un document intitulé «Prise de position de la FMH». Nous vous présentons ici le document de base et la prise de position de la FMH consacrés à l'approche Choosing Wisely.

\begin{abstract}
"Now we're choosing wisely. Body scans, annual exams, can do more harm than good. We're choosing wisely. Imaging for minor things may not give you the truth. We're choosing wisely. Antibiotics for a cold will do nothing but make you ill. We're choosing wisely. A routine screen for many things is often overkill.» (Parodie par James McCormack de la chanson "Happy» de Pharrell Williams, www.youtube.com/watch?v=FqQ-JuRDkl8.)
\end{abstract}

\section{Résumé}

La première campagne "Choosing Wisely" a été lancée en 2012 par la fondation American Board of Internal Medicine (ABIM) dans le but d'éviter les prestations médicales inutiles et d'utiliser de manière responsable les ressources à disposition dans le domaine de la santé. Dans le cadre de cette campagne, des sociétés de discipline médicale de différents pays ont publié des listes "top 5 ", à savoir des listes contenant cinq prestations fréquentes que les médecins et les patients devraient remettre en question. Les campagnes Choosing Wisely visent à réduire les prestations inutiles (surconsommation ${ }^{1}$ ) au moyen de décisions prises en commun entre médecins et patients sur la base de l'évidence scientifique.

Choosing Wisely est une démarche pragmatique émanant du corps médical. Ses points forts résident dans la communication et la sensibilisation aux questions de surconsommation avec une implication ciblée des patients et de la population. Jusqu'à présent, l'efficacité des campagnes Choosing Wisely n'a pas encore fait l'objet d'études. Pour être crédibles, les listes top 5 doivent répondre à des critères qualité.
En 2012, la fondation American Board of Internal Medicine (ABIM) a lancé la première campagne «Choosing Wisely" aux Etats-Unis en posant la question des possibilités d'éviter les prestations médicales inutiles face aux coûts croissants de la santé. Plus de 70 sociétés de discipline médicale des Etats-Unis ont alors publié des listes top 5 regroupant cinq examens ou traitements fréquents que les médecins et les patients devraient remettre en question en raison de leur inutilité ou même de leur caractère nuisible, prouvés par l'évidence scientifique. L'objectif de cette campagne est de sensibiliser la population, les patients et les médecins au thème de la surconsommation et de susciter la discussion entre médecins et patients (shared decision making ou décision partagée ${ }^{2}$ ). Dans ce contexte, les modules de discussion développés pour les médecins (cf. www.choosingwisely.org), le message de la campagne diffusé parmi la population et le matériel d'information pour les patients sur les listes top 5 [4-6], établi avec l'aide de l'organisation de consommateurs "Consumer Reports", ont permis d'inciter les patients à intervenir en tant que partenaires dans la prise de décision. La campagne Choosing Wisely s'est rapidement étendue au niveau international, p. ex. Canada, ${ }^{3}$ Australie, Grande-Bretagne, Italie, Japon et Pays-Bas. Plus près de nous, la Société suisse de médecine interne générale (SSMIG) a publié en 2014 une première liste top 5 intitulée "Smarter Medicine» pour le domaine ambulatoire et en 2016 une deuxième liste pour le secteur hospitalier (cf. www.smartermedicine.ch et Selby et al. [7]). Dans les hôpitaux publics du Tessin, des différences importantes ont été relevées d'un établissement à l'autre concernant cinq pratiques pour lesquelles il existe des recommandations Choosing Wisely [8]. 
En 2015, lors d'une rencontre commune de l'Académie suisse des sciences médicales (ASSM) et de l'Académie suisse pour la qualité en médecine (ASQM), plusieurs experts ont discuté des avantages et des inconvénients des guidelines et de Choosing Wisely $[9,10]$.

Le présent document donne un bref aperçu de Choosing Wisely, ses points forts et ses points faibles, en s'appuyant sur des publications scientifiques (vulgarisées). Il examine en particulier l'efficacité de la démarche et les exigences posées à la méthode. L'objectif est de mettre à la disposition des organisations médicales intéressées et des spécialistes de la santé des informations pour les aider à décider du lancement ou non d'une campagne Choosing Wisely. De plus amples renseignements sur les prestations médicales inutiles figurent dans le document de base de l'ASQM sur la surconsommation [1].

\section{Points forts et points faibles}

Les points forts suivants caractérisent les campagnes Choosing Wisely $[5,6,11]$ :

- Les campagnes émanent du corps médical. Elles montrent que les médecins veulent réfléchir à leurs pratiques et prendre des responsabilités de manière concertée. Grâce à leur mise en œuvre par les sociétés de discipline médicale et les organisations régionales, elles ont un fort potentiel de diffusion.

- Les organisations de consommateurs et de patients sont associées dès le début aux campagnes de diffusion. Des brochures destinées aux patients sont mises librement à leur disposition sur internet. Chaque campagne est communiquée au public via différents médias.

- Les campagnes favorisent la compréhension grâce à leur message simple, clair et limité à cinq recommandations.

- L'objectif consiste à susciter une discussion médecin-patient axée sur la situation individuelle des patients et ne vise aucun rationnement des prestations de santé.

Les campagnes Choosing Wisely ont été critiquées sur les points suivants [6, 11-15]:

- Contrairement aux recommandations, certaines sociétés de discipline médicale ont introduit dans leurs listes top 5 des prestations relevant d'autres disciplines ou ont opté pour des procédures rares et incontestées, donc sans réel impact.

- Aucun échange interdisciplinaire ou interprofessionnel n'a lieu si chaque société de discipline médicale établit seule sa propre liste. Parfois, les listes top 5 ont été rédigées par un petit nombre de per- sonnes sans l'implication des praticiens ou de tous les acteurs concernés et sans la mention des conflits d'intérêts potentiels.

- Peu d'indications ou de recommandations n'existent sur la méthode pour établir une liste top 5 (formulation des objectifs, recherche bibliographique systématique, recherche de consensus ou priorisation). De ce fait, les listes sont très différentes entre elles, elles manquent de transparence quant à la méthode suivie et, pour certaines, rien n'indique que les recommandations sont basées sur l'évidence scientifique.

\section{Effets}

Choosing Wisely part du principe qu'une campagne sensibilise la population et les médecins, qu'elle encourage une prise de décision partagée et diminue ainsi la surconsommation. Une étude [16] a bien montré un lien entre la décision partagée et la surconsommation. Mais ces suppositions doivent être examinées de manière approfondie, notamment par l'évaluation de l'implémentation et de l'effet d'une campagne. Jusqu'à présent, rien ne prouve scientifiquement qu'une campagne Choosing Wisely réduise la surconsommation ou diminue les coûts. Une première étude avec des données administratives [14, 17] n'est pas arrivée à la conclusion que les comportements avaient vraiment changé dans le domaine clinique et elle en a conclu que la publication de listes top 5 ne suffisait pas à elle seule pour éviter la surconsommation. Les initiateurs de Choosing Wisely devraient donc développer des stratégies d'implémentation et tenir compte des différents facteurs susceptibles d'influencer la surconsommation (cf. document de base sur la surconsommation [1]).

\section{Méthode}

Aux Etats-Unis, les initiateurs de la démarche Choosing Wisely ont accordé beaucoup d'autonomie aux sociétés de discipline médicale dans leur manière de procéder afin de les gagner à leur campagne. Ils estiment que les listes top 5 doivent remplir les quatre conditions suivantes [18]:

1) Les recommandations sont basées sur l'évidence scientifique.

2) Le processus d'élaboration est transparent.

3) Elles s'appliquent à des procédures ou tests fréquents.

4) Les prestations citées émanent de leur propre discipline.

Dans une étape suivante, plusieurs voix critiques ont exigé que le développement et la réévaluation des 


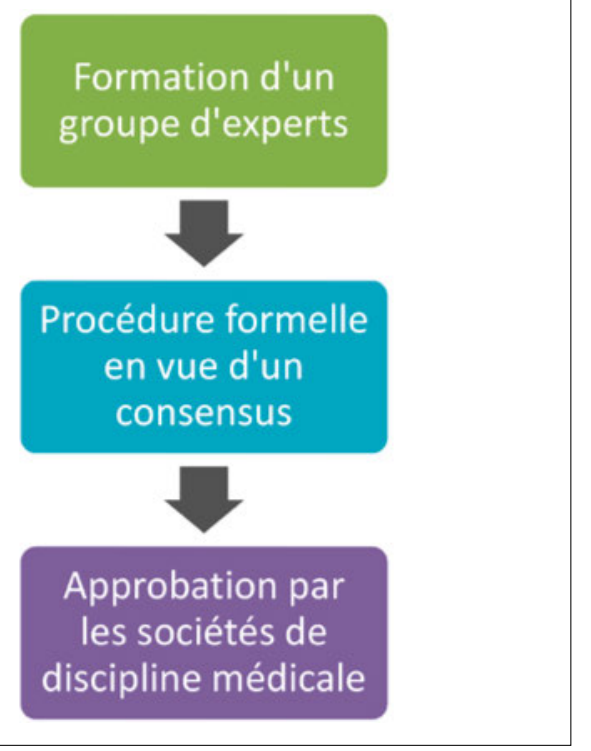

Figure 1: Méthode pour parvenir à un consensus.

listes top 5 se réfèrent à des exemples de bonne pratique (Best practice) ${ }^{4}$ ou à des critères qualité, à l'instar de ce qui est fait avec les guidelines. ${ }^{5}$ Les recommandations suivantes découlent du manuel de la Communauté de travail des sociétés de discipline médicale scientifique allemande [21], en vue d'aider à définir une méthode pour élaborer une liste top 5 .

\section{Consensus}

Un groupe d'experts représentatif des destinataires des recommandations est constitué. Il est composé de médecins des sociétés de discipline médicale concernées, d'autres professionnels de la santé et de représentants d'institutions/organisations impliquées dans les soins, pour autant qu'ils fassent partie des destinataires. Des praticiens, des experts des méthodes scientifiques et idéalement aussi des représentants des patients sont associés aux travaux. Les liens et conflits d'intérêts des participants sont présentés ouvertement.

Pour parvenir à un consensus permettant l'élaboration d'une liste top 5, ce groupe applique une procédure scientifique formelle (p. ex. processus de groupe nominal, conférence consensuelle structurée, technique Delphi). Au cours de la procédure, il s'informe éventuellement aussi auprès d'autres praticiens ou experts [20]. Une fois rédigée, la liste top 5 est adoptée par la société de discipline médicale concernée.

\section{Proposition et choix de recommandations}

Il s'agit tout d'abord de proposer des recommandations pertinentes. Pour cela, il existe différentes manières de procéder: brainstorming entre experts, enquête auprès de praticiens, analyse des publications scientifiques, guidelines (recommandations Do not) et listes Choosing Wisely existantes $[5,7,16]$.
4 Dans JAMA Internal Medicine [15], Schuur et al. [20] sont cités en exemple pour leur méthode.

5 Cf. AGREE II [19]

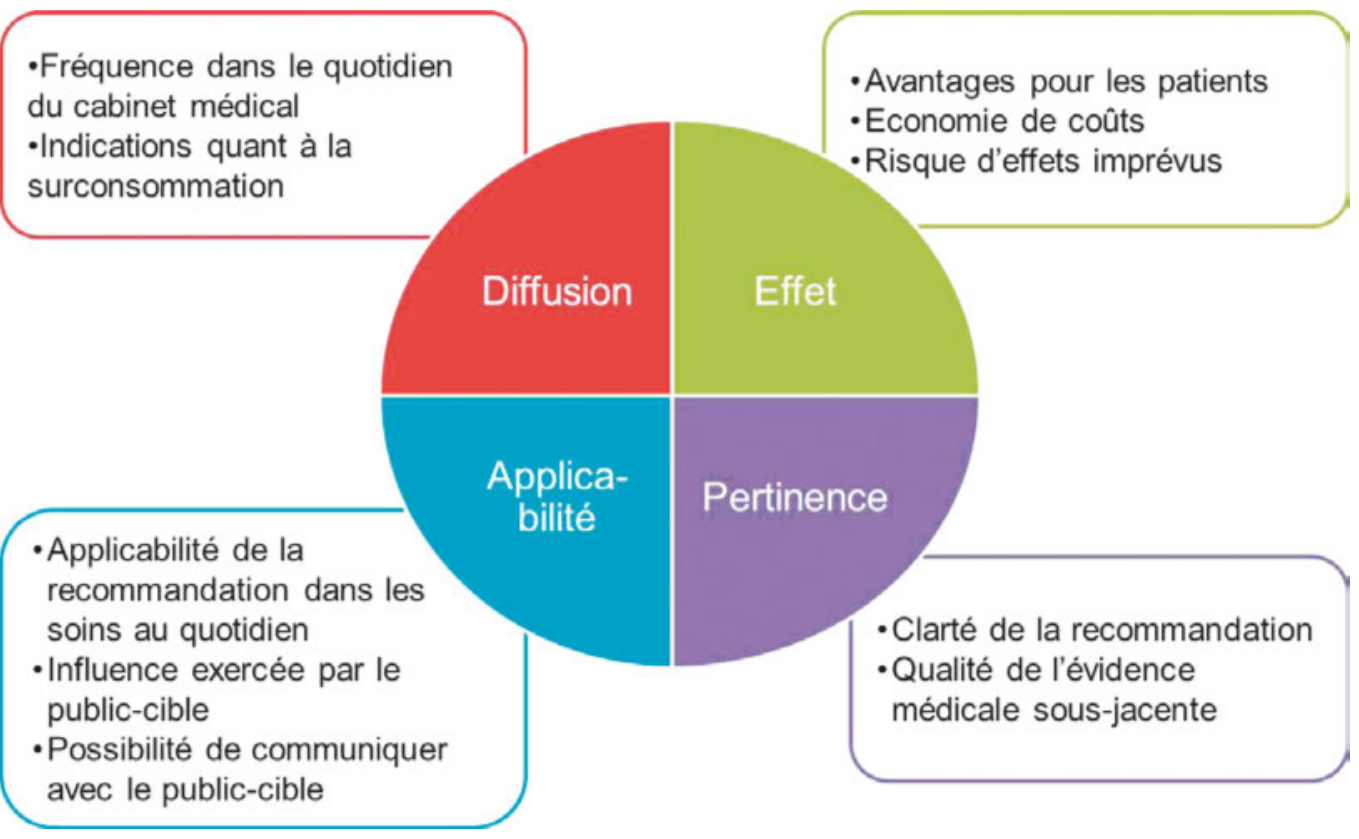

Figure 2: Critères qualité envisageables pour choisir les recommandations Choosing Wisely (représentation basée sur [7, 19-21]). 
Ensuite, les recommandations obtenues sont évaluées en fonction de critères explicites qui peuvent être répartis en quatre domaines comme le montre la figure 2 ci-après.

\section{Diffusion et implémentation}

- Fixer les objectifs d'une campagne Choosing Wisely

- Définir la stratégie pour la diffusion parmi les professionnels de la santé, les patients, les autorités et le public

- Rédiger le matériel d'information pour les patients et les modules de discussion pour les médecins

- Mettre en œuvre la campagne en association avec les cliniques et les cabinets médicaux

- Planifier l'évaluation

- Planifier la mise à jour

\section{Discussion}

Les campagnes Choosing Wisely sont un premier pas pour réduire la surconsommation, avec l'objectif de sensibiliser les patients et les médecins et de modifier leur comportement. Elles soutiennent les médecins dans la discussion avec leurs patients sur les prestations inutiles. Choosing Wisely a été développé en vue d'une répartition juste des ressources de la santé, mais peut également viser des améliorations de la qualité. De ce fait, Choosing Wisely peut aussi être une réponse possible aux questions de surconsommation (cf. document de base sur la surconsommation [1]). C'est une approche pragmatique à laquelle il manque cependant des critères qualité et la transparence sur la méthode. Jusqu'à présent, aucune évaluation des campagnes Choosing Wisely n'a été effectuée, si bien qu'il n'existe encore aucun élément prouvant leur efficacité. Il semble cependant que la seule diffusion de listes top 5 ne suffise pas, à l'instar des guidelines [22], pour une mise en œuvre efficace des recommandations dans la pratique.

\section{Références}

1 Gerber M, Kraft E, Bosshard C. La surconsommation de prestations médicales: un problème de qualité. Bulletin des médecins suisses. 2016;97(7):236-43.

2 Wirtschaftslexikon.co. Überversorgung. 2015 Accessed: 15.8.2016, Available from: http://www.wirtschaftslexikon.co/d/ ueberversorgung/ueberversorgung.htm.
3 Gerber M, Kraft E, Bosshard C. Décision partagée - Médecin et patient décident ensemble. Bulletin des médecins suisses. 2014;95(50):1883-9.

4 ABIM Foundation. Choosing Wisely. Accessed: 23.10.2016, Available from: http://www.choosingwisely.org.

5 Morden NE, Colla CH, Sequist TD, Rosenthal MB. Choosing wisely - the politics and economics of labeling low-value services. $\mathrm{N}$ Engl J Med. 2014;370(7):589-92.

6 Admon AJ, Cooke CR. Will Choosing Wisely ${ }^{\circledR}$ Improve Quality and Lower Costs of Care for Patients with Critical Illness? Ann Am Thorac Soc. 2014;11(5):823-7.

7 Selby K, Gaspoz JM, Rodondi N, Neuner-Jehle S, Perrier A, Zeller A, et al. Creating a list of low-value health care activities in Swiss primary care. JAMA Intern Med. 2015;175(4):640-2.

8 Gabutti L, Pironi M, Della Bruna R, Heinkel J, Clivio L, Greco A. Choosing Wisely: von der Theorie zur Praxis. Rev Med Suisse. 2015;11(491):1973-7.

9 Amstad H, Gaspoz J-M, Zemp L. Guidelines und Choosing wisely: to do's and not to do's. Bulletin des médecins suisses. 2015;96(5):130-1.

10 Kesseli B. Wertvoll, aber aufwendig. Impressionen vom Workshop "Choosing wisely und Guidelines: Chancen und Stolpersteine». Bulletin des médecins suisses. 2015;96(26):966-9.

11 O'Callaghan G, Meyer H, Elshaug AG. Choosing wisely: the message, messenger and method. Med J Aust. 2015;202(4):175-7.

12 Malhotra A, Maughan D, Ansell J, Lehman R, Henderson A, Gray M, et al. Choosing Wisely in the UK: the Academy of Medical Royal Colleges' initiative to reduce the harms of too much medicine. Bmj. 2015;350:h2308.

13 Strech D, Follmann M, Klemperer D, Lelgemann $M$, Ollenschläger $\mathrm{G}$, Raspe $\mathrm{H}$, et al. When Choosing Wisely meets clinical practice guidelines. Z. Evid. Fortbild. Qual. Gesundh.wesen (ZEFQ) 2014;108:601-3.

14 Rosenberg A, Agiro A, Gottlieb M, Barron J, Brady P, Liu Y, et al. Early trends among seven recommendations from the Choosing Wisely campaign. JAMA Intern Med. 2015;175(12).

15 Grady D, Redberg RF, Mallon WK. How should top-five lists be developed? What is the next step? JAMA Intern Med. 2014;174(4).

16 Légaré F, Labrecque M, Cauchon M, Castel J, Turcotte S, Grimshaw J. Training family physicians in shared decision-making to reduce the overuse of antibiotics in acute respiratory infections: a cluster randomized trial. Cmaj. 2012;184(13):E726-34.

17 Gonzales R, Cattamanchi A. Changing clinician behavior when less is more. JAMA Intern Med. 2015;175(12).

18 Baron RJ, Wolfson D. Advancing medical professionalism and the choosing wisely campaign. JAMA Intern Med. 2015;175(3):464-5.

19 Brouwers M KM, Browman GP, Cluzeau F, Feder G, Fervers B, Hanna S, Makarski J on behalf of the AGREE Next Steps Consortium. AGREE II: Advancing guideline development, reporting and evaluation in healthcare. Deutsche Übersetzung. 2014. Available from: http://www.agreetrust.org/wp-content/uploads/2014/03/ AGREE_II_German-Version.pdf.

20 Schuur JD, Carney DP, Lyn ET, Raja AS, Michael JA, Ross NG, et al. A top-five list for emergency medicine a pilot project to improve the value of emergency care. JAMA Intern Med. 2014;174(4):509-15.

21 Arbeitsgemeinschaft der Wissenschaftlichen Medizinischen Fachgesellschaften (AWMF) - Ad hoc Kommission «Gemeinsam klug Entscheiden". Manual Entwicklung von Empfehlungen im Rahmen der Initiative Gemeinsam Klug Entscheiden. Version 1.1. 2016. Available from: http://www.awmf.org/medizin-versorgung/gemeinsam-klug-entscheiden.html.

22 Prior M, Guerin M, Grimmer-Somers K. The effectiveness of clinical guideline implementation strategies - a synthesis of systematic review findings. J Eval Clin Pract. 2008;14(5):888-97. 